\title{
The blood/cerebrospinal fluid partitioning of pyrazinamide: a study during the course of treatment of tuberculous meningitis
}

\author{
Prida Phuapradit, K Supmonchai, S Kaojarern, C Mokkhavesa
}

\begin{abstract}
Concentrations of pyrazinamide were measured in serum and cerebrospinal fluid (CSF) of 17 adult patients with tuberculous meningitis up to six months after starting treatment. Pyrazinamide penetrated excellently into the CSF and mean concentrations at various intervals up to six months of treatment were consistently above that required for inhibition of the growth of Mycobacterium tuberculosis. The blood/CSF partitioning of pyrazinamide does not change as the patients recover from the meningitis.
\end{abstract}

Pyrazinamide has been used increasingly in recent years for treatment of tuberculous meningitis. ${ }^{2}$ The excellent penetration of pyrazinamide into the cerebrospinal fluid (CSF) was first demonstrated in a single case report of a patient with tuberculous meningitis. $^{3}$ This finding was confirmed in recent studies in a larger number of patients with tuberculous meningitis. ${ }^{4-6} \mathrm{~A}$ number of antituberculosis drugs, notably ethambutol, rifampicin and streptomycin, pass into the CSF in sufficient concentrations during the active stage of tuberculous meningitis but, after treatment, as the blood-brain barrier is restored, the concentrations of these drugs in the CSF decrease. ${ }^{7}$ Data on the penetration of pyrazinamide into the CSF during the course of treatment of the meningitis are limited. We conducted a study to measure the levels of pyrazinamide the serum and CSF of 17 adult patients with tuberculous meningitis at various intervals up to six months after starting treatment.

\section{Patients and Methods}

The ages of the 17 patients ranged from 15 to 70 years with a mean of 33 years. All patients presented with lymphocytic meningitis with low CSF glucose and raised CSF protein concentrations. M tuberculosis was grown from CSF in four patients. Eleven patients had associated active pulmonary tuberculosis, which in six was miliary. Caseating cervical lymphadenitis was found in three patients.
Thirteen patients belonged to the previously reported series of 28 patients who received oral pyrazinamide $1500 \mathrm{mg}(30 \mathrm{mg} / \mathrm{kg}$ for those who weighed less than $50 \mathrm{~kg}$ ), rifampicin 600 $\mathrm{mg}$ (450 mg for those who weighed less than 45 $\mathrm{kg}$ ), isoniazid $300 \mathrm{mg}$ and intramuscular streptomycin 750-1000 mg daily as single doses for two months, followed by isoniazid $300 \mathrm{mg}$ and rifampicin $600 \mathrm{mg}$ daily for seven months. ${ }^{1}$ The other four patients received similar doses of pyrazinamide, rifampicin and isoniazid $300 \mathrm{mg}$ as single daily doses for six months with streptomycin $1000 \mathrm{mg} /$ day by intramuscular injection during the first two months. Lumbar punctures were periodically performed to assess response to treatment. The CSF and serum were collected three hours after a $1500 \mathrm{mg}$ oral single dose of pyrazinamide at various intervals of the treatment.

Pyrazinamide was assayed spectrophotometrically by the method described by Gurumurthy et al. ${ }^{8}$ The CSF and serum levels of pyrazinamide during the first five weeks of treatment were obtained in 13 patients. In four patients the levels were measured at the 25th week of the treatment. The concentrations of pyrazinamide in the serum and CSF were presented as means and standard deviations. Correlation between the serum and CSF levels of all intervals was analysed by linear regression. Mean ratios of the CSF and serum concentrations of pyrazinamide at various intervals were calculated and compared by analysis of variance.

\section{Results}

There were 41 paired specimens of CSF and serum taken at various intervals of the treatment. Mean concentrations and standard deviations of pyrazinamide in the CSF and serum are shown in the table. There was a good correlation between the concentrations of pyrazinamide in the serum and CSF $(P<$ $0.001)$ with a correlation coefficient $(r)$ of $0.8701(\mathrm{n}=41)$ (fig). Analysis of variance showed no statistical difference in the CSF/ serum ratios of pyrazinamide concentrations at different times from starting treatment at the 0.050 level. 
Table Mean concentrations (SD) of pyrazinamide concentrations in the serum and CSF

\begin{tabular}{|c|c|c|c|c|c|c|c|}
\hline \multirow{2}{*}{$\begin{array}{l}\text { Weeks of } \\
\text { treatment }\end{array}$} & \multirow{2}{*}{$\begin{array}{l}\text { Number of } \\
\text { paired } \\
\text { samples of } \\
\text { serum and } \\
C S F\end{array}$} & \multicolumn{4}{|c|}{ Mean concentration $(\mu \mathrm{g} / \mathrm{ml})(S D)$} & \multirow{2}{*}{\multicolumn{2}{|c|}{$\begin{array}{l}\text { CSF/serum } \\
\text { ratio }\end{array}$}} \\
\hline & & \multicolumn{2}{|c|}{ Serum } & \multicolumn{2}{|c|}{$C S F$} & & \\
\hline $\begin{array}{r}1 \\
2 \\
3 \\
4 \\
5 \\
25\end{array}$ & $\begin{array}{r}11 \\
13 \\
5 \\
4 \\
4 \\
4\end{array}$ & $\begin{array}{l}42 \cdot 3 \\
32 \cdot 2 \\
39 \cdot 9 \\
49 \cdot 0 \\
35 \cdot 5 \\
38 \cdot 1\end{array}$ & $\begin{array}{r}(17.2) \\
(11.2) \\
(11.8) \\
(16.7) \\
(6.1) \\
(6.6)\end{array}$ & $\begin{array}{l}39 \cdot 7 \\
29.5 \\
33.6 \\
50 \cdot 2 \\
37 \cdot 2 \\
29.7\end{array}$ & $\begin{array}{r}(14 \cdot 2) \\
(12 \cdot 0) \\
(8 \cdot 2) \\
(14 \cdot 6) \\
(7 \cdot 6) \\
(6 \cdot 2)\end{array}$ & $\begin{array}{l}0.95 \\
0.92 \\
0.87 \\
1.04 \\
1.05 \\
0.79\end{array}$ & $\begin{array}{l}(0.18) \\
(0 \cdot 18) \\
(0.17) \\
(0.06) \\
(0.03) \\
(0.17)\end{array}$ \\
\hline
\end{tabular}

Figure Plot of CSF concentrations of pyrazinamide against the serum concentrations for 17 patients with tuberculous meningitis.

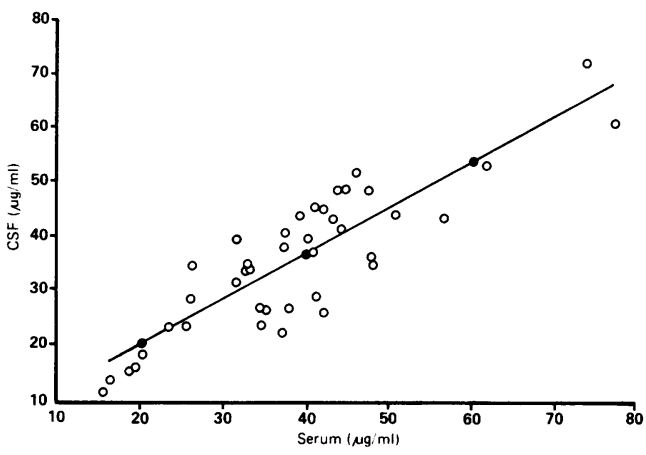

Discussion

Our study confirmed excellent penetration of pyrazinamide into the CSF in all the patients and that blood/CSF partitioning of pyrazinamide did not change as the patients recovered. The mean concentrations of pyrazinamide in the CSF, measured at various intervals up to six months of the treatment, were consistently above that required for inhibition of the growth of $M$ tuberculosis. ${ }^{910}$ There were no statistical differences between the CSF/serum ratios at various intervals of the study. Even at six months of the treatment therapeutic concentrations of pyrazinamide in the CSF were still maintained. Further studies on the lipid solubility and protein binding of pyrazinamide may explain its excellent entry into the CSF.

In view of its potent sterilising antituberculous activity, reasonable cost, low incidence of major adverse effects ${ }^{111}$ and excellent CSF pharmacokinetic property, pyrazinamide should be included in the regimen of drugs used in the treatment of tuberculous meningitis.

1 Phuapradit P, Vejiajiva A. Treatment of tuberculous meningitis: role of short-course chemotherapy. $Q J$ Med 1987; $62 \cdot 249-58$

2 Pauranik A, Behari M, Maheshwari MC. Pyrazinamide in treatment of tuberculous meningitis. Arch Neurol 1986; 43:982.

3 Forgan-Smith R, Ellard G, Newton D, Michison DA. Pyrazinamide and other drugs in tuberculous meningitis. Lancet 1973;ii:374.

4 Supmonchai K. Studies of cerebrospinal fluid and serum levels of antituberculous drugs in tuberculous meningitis. MS Thesis, Chulalongkorn University, Bangkok, 1985.

5 Ellard GA, Humphries MJ, Gabriel M, Teoh R. Penetration of pyrazinamide into cerebrospinal fluid in tuberculous meningitis. Br Med J 1987;294:284-5.

6 Donald PR, Seifart H. Cerebrospinal fluid pyrazinamide concentrations in children with tuberculous meningitis. concentrations in children with tub

7 Holdiness MR. Cerebrospinal fluid pharmacokinetics of antituberculosis drugs. Clin Pharmacokinet 1985;10: antituberc

8 Gurumurthy P, Nair NGK, Raghupati SG. Methods for estimation of pyrazinamide and pyrazinoic acid in body fluids. Indian J Med Res 1980;71:129-34.

9 Stottmeier KD, Beam RE, Kubika GP. Determination of drug susceptibility of mycobacteria to pyrazinamide in 7 H10 agar. Am Rev Respir Dis 1967;96:1072-5

10 Carlone NA, Acocella G, Cuffini AN, Forno-Pizzoglio M. Killing of macrophage-ingested mycobacteria by rifampicin, pyrazinamide, pyrazinoic acid alone and in combination. Am Rev Respir Dis 1985;132:1274-7.

11 Donald PR, Schoeman JF, Kennedy A. Hepatic toxicity during chemotherapy for severe tuberculous meningitis. Am J Dis Child 1987;141:741-3. 